The mean levels of IgG aß2-GP-1, LA, aOxLDL in SLE patients and mean levels of $L A, a O x L D L$ in RA patients were above standard values but were similar in both groups. Moreover, the mean levels of IgG aCL, IgM aß2-GP-1, IgG/lgM aAnV and aPT were comparable in patients with SLE and RA. However, the mean levels of IgM aCL in RA patients was higher than in SLE patients. This may indicate an increased autoimmune activity.

Simultaneous elevation in four types of aPL levels was observed in $4,4 \%$ patients with RA, in 2,5\% - with SLE; simultaneous elevation in three types of aPL was reveled in $11,1 \%$ patients with RA, in $17,5 \%$ - with SLE; two types of aPL were increased in $35,6 \%$ patients with RA and $27,5 \%$ - with SLE. Only one type of elevated aPL levels $(12,9 \%)$ was indentified in the control group. Conclusion: Thus, patients with RA are characterized by a wide range of aPL. Qualitative and quantitative changes in the levels and types of autoantibodies in patients with RA have been established similar to those in SLE patients. In patients with autoimmune rheumatic pathology, the presence of simultaneously elevated several types of aPL have been proved.

Disclosure of Interests: None declared

DOI: 10.1136/annrheumdis-2020-eular.5796

\begin{tabular}{|l|l|}
\hline AB0088 & N6-METHYLADENOSINE-MODIFIED CIRC_0088194 \\
PROMOTES MIGRATION AND INVASION OF \\
RHEUMATOID ARTHRITIS FIBROBLAST-LIKE \\
SYNOVIOCYTES THROUGH MIR-766-3P/MMP2 AXIS
\end{tabular}

Y. Cai ${ }^{1,2}$, M. Yang ${ }^{3}$, Q. Ouyang ${ }^{4}$, Q. Huang ${ }^{4}$, R. Liang ${ }^{2,4} .{ }^{1}$ Nanfang Hospital, Southern Medical University, Department of Rheumatology and Immunology, Guangzhou, China; ${ }^{2}$ Southern Medical University, Guangzhou, China; ${ }^{3}$ Nafang Hospital, Southern Medical University, Department of Rheumatology and Immunology, Guangzhou, China; ${ }^{1}$ Nanfang Hospital, Southern Medical University, Department of Rheumatology and Immunology, Guangzhou, China

Background: Circular RNAs (circRNAs) participate in the initiation and progression of various diseases by miRNA sponges including postmenopausal osteoporosis[1], bladder cancer[2], and osteoarthritis (OA)[3]. However, the activity of circRNAs as "miRNA sponges" in rheumatoid arthritis (RA) has not been studied.

Objectives: To investigate whether circRNA acts as competing endogenous RNA to regulate pathological processes of RA, and whether the N6-methyladenosine $\left(\mathrm{m}^{6} \mathrm{~A}\right)$ modification affects Circ_0088194 stability in RA fibroblast-like synoviocytes (RA-FLSs).

Methods: CircRNA microarray analysis was conducted to characterize the expression profiles of circRNAs in 3 RA-FLSs and 3 osteoarthritis fibroblast-like synoviocytes (OA-FLSs). Methylated RNA immunoprecipitation was performed to validate the level of $\mathrm{m}^{6} \mathrm{~A}$ modification on Circ_0088194 in RA-FLSs and OA-FLSs. Dual-luciferase reporter assay, bioinformatics analysis, protein array analysis, and fluorescence in situ hybridization were employed to evaluate the interaction between Circ_0088194 and miR-766-3p, and between target miR766-3p and matrix metalloproteinase 2 (MMP2).

Results: Overexpression of Circ_0088194 promoted the migration and invasion of RA-FLSs and increased MMP2 expression. The expression and function of miR-766-3p were inversely correlated with Circ_0088194, which sponged miR-766-3p to upregulate MMP2 expression. Although $m^{6} A$ modification of Circ_0088194 exists in RA-FLSs and OA-FLSs, their level did not differ.

Conclusion: This study presents an important role of this novel circRNA as a sponge of miR-766-3p to promote RA-FLS migration and invasion by targeting MMP2. However, the modification might not affect Circ_0088194 stability in RA-FLSs and OA-FLSs. Therefore, Circ_0088194 may contribute to RA development and represent as an auspicious therapeutic target.

References:

[1] Yu, L., and Liu, Y. (2019). circRNA_0016624 could sponge miR-98 to regulate BMP2 expression in postmenopausal osteoporosis. Biochem Biophys Res Commun 516: 546-550.

[2] Yang, C., et al. (2018). Circular RNA circ-ITCH inhibits bladder cancer progression by sponging miR-17/miR-224 and regulating p21, PTEN expression. MOL CANCER 17.

[3] Shen, S., et al. (2019). CircSERPINE2 protects against osteoarthritis by targeting miR-1271 and ETS-related gene. ANN RHEUM DIS 78: 826-836.

Disclosure of Interests: None declared

DOI: 10.1136/annrheumdis-2020-eular.340

\section{AB0089 THE ANALYSIS FOR THE INHIBITION OF ANGIOGENESIS BY JAK INHIBITOR}

M. Fujita ${ }^{1}$, K. Fukuda ${ }^{1}$, S. Hayashi ${ }^{1}$, K. Kikuchi ${ }^{1}$, Y. Takashima ${ }^{1}$, T. Kamenaga ${ }^{1}$, T. Maeda ${ }^{2}$, T. Matsubara ${ }^{1}$, R. Kuroda ${ }^{1} .{ }^{1}$ Kobe University Graduate School of Medicine, Orthopaedic Surgery, Kobe, Japan; ${ }^{2}$ Matsubara Mayflower Hospital, Orthopaedic Surgery, Kato, Japan
Background: Many blood vessels are generated in the hyperplastic synovial tissue of patients with rheumatoid arthritis (RA), and lead to chronic tissue inflammation and joint destruction [1]. Janus kinase (JAK) family consisting of JAK1, JAK2, JAK3 and tyrosine kinase 2 (TYK2) are chain receptors which phosphorylate signal transducers and activators of transcription (STAT) and mediate inflammatory diseases including RA [2]. Nowadays, several JAK inhibitors such as Tofacitinib (TOF), Baricitinib (BAR) and Peficitinib (PEF) have been developed and demonstrated to have the inhibitory effects on inflammatory arthritis [3-5]. However, there were few reports concerning their effects on angiogenesis in vitro. Objectives: The purpose of the present study is to investigate the influence of JAK inhibitors on angiogenesis of human umbilical vein endothelial cell (HUVEC) activated by vascular endothelial growth factor (VEGF).

Methods: The cell line of HUVECs were used for this study. The activity of proliferation and tube formation were analyzed by counting assay and tube formation assay, respectively.

In counting assay, HUVECs $\left(5 \times 10^{4} \mathrm{cells} / \mathrm{ml}\right)$ were seeded onto 96 -well cell culture plate with $20 \mathrm{ng} / \mathrm{ml}$ VEGF including various doses $(0.1 \mu \mathrm{M}, 1 \mu \mathrm{M}, 5 \mu \mathrm{M})$ of TOF, BAR or PEF. After 48 hours incubation at $37^{\circ} \mathrm{C}$ in a humidified atmosphere containing $5 \% \mathrm{CO}_{2}$, cell proliferation of each groups was assessed using cell counting kit. In tube formation assay, HUVECs $\left(5 \times 10^{4}\right.$ cells $\left./ \mathrm{ml}\right)$ were treated with $20 \mathrm{ng} / \mathrm{ml}$ VEGF including various dose $(0.1 \mu \mathrm{M}, 1 \mu \mathrm{M}, 5 \mu \mathrm{M})$ of TOF, BAR or PEF for 00 hours, then seeded onto 48-well plate applied with Matrigel. After 24 hours incubation on Matrigel, the capillary-like tube formation of each well was photographed using phase contrast microscopy. Tube formation were quantitated by measurement of the length of branch.

Results: HUVECs were activated in proliferation and tube formation by VEGF treatment. And, the proliferation and tube formation of HUVECs activated by VEGF were suppressed by All of TOF, BAR and PEF. In particular, TOF and PEF could suppress them highly.

Conclusion: This study showed the inhibitory effect of JAK inhibitors on proliferation and tube formation of HUVECs activated by VEGF. In particular, the angiogenesis of HUVECs activated by VEGF was highly suppressed by TOF and PEF. VEGF is reported to regulate the angiogenesis through multi JAK-STAT signaling pathways [6]. The inhibitory effects on angiogenesis of TOF, BAR and PEF might depend on the differences in their affinity for JAKs. VEGF has been shown to a have a central involvement in the angiogenic process in RA [7]. JAK inhibitors might suppress the angiogenesis in RA synovial tissues by inhibiting VEGF signaling.

References:

[1] Scott DL, et al. Rheumatoid arthritis. Lancet. 2010.

[2] Banerjee S, et al. JAK-STAT signaling as a target for inflammatory and autoimmune diseases: current and future prospects. Drugs. 2017.

[3] William D, et al. JAK inhibitors in dermatology: the promise of a new drug class. Journal of the American Academy of Dermatology. 2017.

[4] Dhillon S. Tofacitinib: A Review in Rheumatoid Arthritis. Drugs. 2017.

[5] Markham A, et al. Peficitinib: First Global Approval. Drugs. 2019.

[6] Zhang HY, et al. Three important components in the regeneration of the cavernous nerve: brain-derived neurotrophic factor, vascular endothelial growth factor and the JAK/STAT signaling pathway. Asian journal of andrology. 2011

[7] Paleolog EM. Angiogenesis in rheumatoid arthritis. Arthritis research. 2002.

Acknowledgments: none

Disclosure of Interests: None declared

DOI: 10.1136/annrheumdis-2020-eular.3161

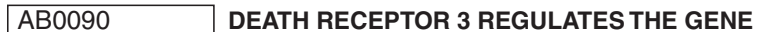 EXPRESSIONS OF VARIOUS KEY MOLECULES IN RHEUMATOID SYNOVIAL FIBROBLASTS}

K. Fukuda ${ }^{1}$, Y. Miura ${ }^{1,2}$, S. Hayashi ${ }^{1}$, T. Maeda ${ }^{1}$, R. Kuroda ${ }^{1} .{ }^{1}$ Kobe University Graduate School of Medicine, Department of Orthopaedic Surgery, Kobe, Japan; ${ }^{2}$ Kobe University Graduate School of Health Sciences, Division of Orthopedic Science, Department of Rehabilitation Science, Kobe, Japan

Background: Rheumatoid arthritis (RA) is a chronic inflammatory disease that causes hyperplasia of synovial tissue. Death receptor 3 (DR3) is a tumor necrosis factor receptor and binds to TL1A, a member of the TNF family. DR3 is involved in the mechanism of cell proliferation and apoptosis through NF-KB signaling. Suppression of DR3 in rheumatoid synovial fibroblasts (RA-FLS) is associated with hyperplasia of rheumatoid synovial tissue [1]. We previously revealed the expression profiles regulated by TL1A, suggesting that TL1A might affect the pathogenesis of RA, including proliferation, regulation of $B$ cells and $T$ cells, inflammation, and cytokine processing [2]

Objectives: In this study, we investigated the gene expression profiles regulated by DR3 in RA-FLS to reveal how DR3 is involved in the pathogenesis of RA.

Methods: RA-FLS were from patients with RA. Four individual lines of primary cultured RA-FLS were incubated either with $1000 \mathrm{ng} / \mathrm{ml}$ of human DR3-Fc 